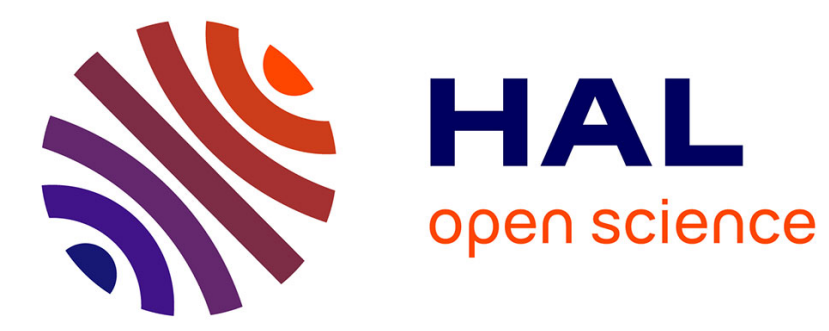

\title{
First astrophysical results from AMBER/VLTI
}

Fabien Malbet, Romain G. Petrov, Gerd Weigelt, Philippe Stee, Eric Tatulli, Armando Domiciano de Souza, Florentin Millour

\section{To cite this version:}

Fabien Malbet, Romain G. Petrov, Gerd Weigelt, Philippe Stee, Eric Tatulli, et al.. First astrophysical results from AMBER/VLTI. Advances in Stellar Interferometry, 2006, Orlando, United States. hal00080756

\section{HAL Id: hal-00080756 https://hal.science/hal-00080756}

Submitted on 20 Jun 2006

HAL is a multi-disciplinary open access archive for the deposit and dissemination of scientific research documents, whether they are published or not. The documents may come from teaching and research institutions in France or abroad, or from public or private research centers.
L'archive ouverte pluridisciplinaire HAL, est destinée au dépôt et à la diffusion de documents scientifiques de niveau recherche, publiés ou non, émanant des établissements d'enseignement et de recherche français ou étrangers, des laboratoires publics ou privés. 


\title{
First astrophysical results from AMBER/VLTI
}

\author{
F. Malbet ${ }^{a}$, R.G. Petrov ${ }^{b}$, G. Weigelt ${ }^{c}$, P. Stee $^{d}$, E. Tatullie ${ }^{e}$ A. Domiciano de Souza ${ }^{b}, d$, F. \\ Millour $^{a, b}$ and the AMBER consortium \\ ${ }^{a}$ Laboratoire d'Astrophysique de Grenoble, BP 53, F-38041 Grenoble cedex 9, France; \\ ${ }^{b}$ Laboratoire Universitaire d'Astrophysique de Nice, France; \\ ${ }^{c}$ Max-Planck Institut für Astrophysick, Bonn, Germany; \\ ${ }^{d}$ Observatoire de la Côte d'Azur, Nice, France; \\ ${ }^{e}$ Osservatorio Astrofisico di Arcetri, Firenze, Italy
}

\begin{abstract}
The AMBER instrument installed at the Very Large Telescope (VLT) combines three beams from as many telescopes to produce spectrally dispersed fringes with milli-arcsecond angular scales in the near infrared. Two years after installation, first scientific observations have been carried out during the Science Demonstration Time and the Guaranteed Time mostly on bright sources due to some VLTI limitations. In this paper, we review these first astrophysical results and we show which types of completely new information is made available by AMBER.

The first astrophysical results have been mainly focusing on stellar wind structure, kinematics, and its interaction with dust usually concentrated in a disk. Because AMBER has dramatically increased the number of measures per baseline, this instrument brings strong constraints on morphology and models despite a relatively poor $(u, v)$ coverage for each object.
\end{abstract}

Keywords: Optical interferometry, infrared, young stellar objects, stars, evolved stars, massive stars, hot stars

\section{INTRODUCTION}

AMBER is one of the first-generation instruments of the Very Large Telescope Interferometer (VLTI) that has been described by Petrov et al. ${ }^{1,2}$ The science program prepared has already partly been described by Malbet et al. ${ }^{3,4}$ in previous SPIE papers.

AMBER is an interferometric beam combiner for the VLTI working in the near-infrared $J, H, K$ bands. It is able to simultaneously handle 3 beams coming from 3 identical telescopes. AMBER interferograms are spectrally dispersed with a resolution of about 35, 1200 and 10000. Therefore AMBER can measure vibilities and a closure phase in a few hundred different spectral channels.

The spectral coverage, the spectral resolution and the better sensitivity compared to small-aperture interferometers give access to many new astrophysical fields that we describe preliminaryly in this review.

\section{WIND/DISK CONNECTION IN YOUNG STARS OF INTERMEDIATE MASSES}

The young stellar object MWC 297 is an embedded Herbig Be star exhibiting strong hydrogen emission lines and a strong near-infrared continuum excess which has been observed with the AMBER instrument during its first commissioning run. ${ }^{5}$ MWC 297 has been spatially resolved in the continuum with a visibility of $\sim 0.50$ as well as in the $\operatorname{Br} \gamma$ emission line where the visibility decreases to a lower value of $\sim 0.33$. A first interpretation of this result is that the gas emitting the $\operatorname{Br} \gamma$ emission line is located in a region slightly larger than the one from which the dust continuum emission arises.

A picture emerges in which MWC 297 is surrounded by an equatorial flat disk that is possibly still accreting and by an outflowing wind. AMBER's unique capability to measure spectral visibilities allowed Malbet et al. ${ }^{5}$ for the first time to compare the apparent geometry of a wind with the disk structure in a young stellar

Further author information: (Send correspondence to F. Malbet)

E-mail: fabien.malbet@obs.ujf-grenoble.fr 


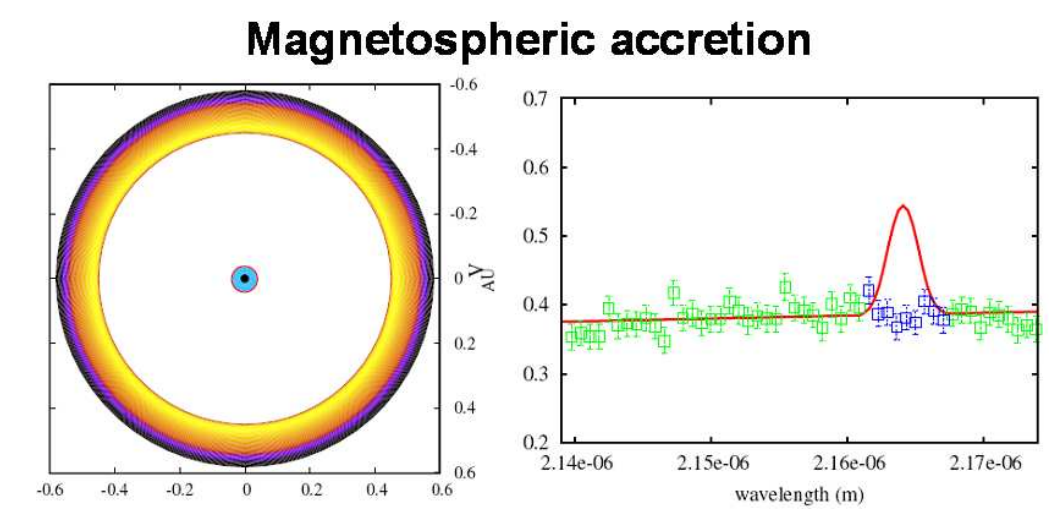

\section{Outflowing wind}
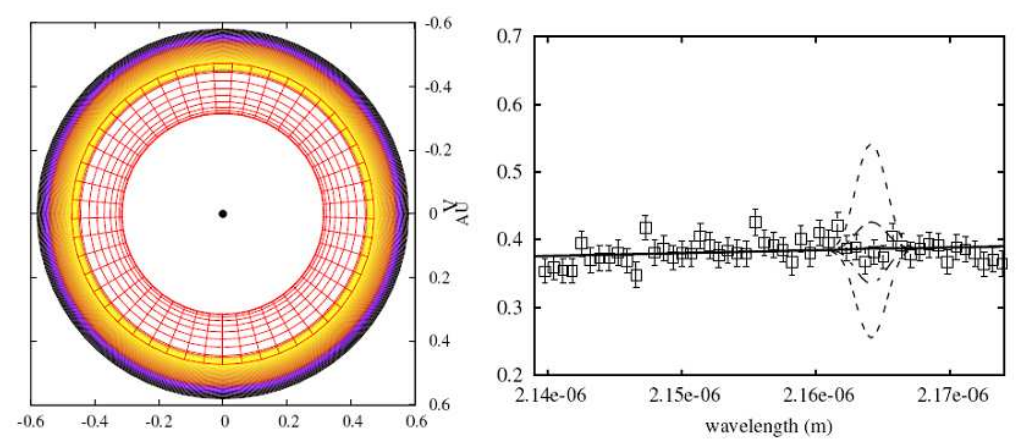

Figure 1. Model (left part) and observation (right part) of the young stellar object HD 104237. The upper panel shows, in solid line, the result of a simulation of the gas emission if it would come from the magnetosphere. The bottom panel shows in solid line the result from a simulation of an outflowing wind. The dashed line corresponds to different sizes of the wind launching region. The best fit corresponds to a launching region between 0.3 and 0.5AU.

system. However the measurements were not sufficient to unambiguously choose between the different scenarios of wind-launching region: disk wind or X-wind.

A lower mass, less active system, the Herbig Ae system HD104237 has also been observed with AMBER. ${ }^{6}$ The central A2 emission line star is surrounded by a circumstellar disk, which causes the infrared excess emission and drives a jet seen in Ly- $\alpha$ images. The optical spectrum shows a rather narrow $\mathrm{H} \alpha$ emission with a $\mathrm{P}-\mathrm{Cygni}$ profile. The visibility measured by AMBER does not vary between the continuum and the Br $\alpha$ line, even though the line is strongly detected in the spectrum, with a peak intensity $35 \%$ above the continuum (see Fig. 1). This demonstrates that the line and continuum emission have similar size scales. Assuming that the K-band continuum excess originates in a puffed-up inner rim of the circumstellar disk, Tatulli et al. ${ }^{6}$ conclude that this emission most likely arises from a compact disk wind very close to the inner rim location.

These two results show that AMBER on the VLTI is going to be a major tool for understanding the very close environment of young stars and will disentangle the region of emission from dust and gas, especillay coming from the disk and the wind.

\section{ROTATING GAS ENVELOPE AROUND HOT BE AND B[E] STARS}

Several emission-line supergiants have been scrutinized by AMBER: $\alpha$ Arae, one of the closest Be stars, ${ }^{7} \kappa$ Canis Majoris, one of the brightest ones ${ }^{8}$ and CPD- $57^{\circ} 2874$ a B $[\mathrm{e}]$ supergiant star characterized by dust emission. ${ }^{9}$

The AMBER instrument operating in the $K$ band provides a gain by a factor of 5 in spatial resolution compared to previous VLTI/MIDI observations of $\alpha$ Arae. Moreover, it is possible to combine the high angular resolution provided with the (medium) spectral resolution of AMBER to study the kinematics of the inner part 


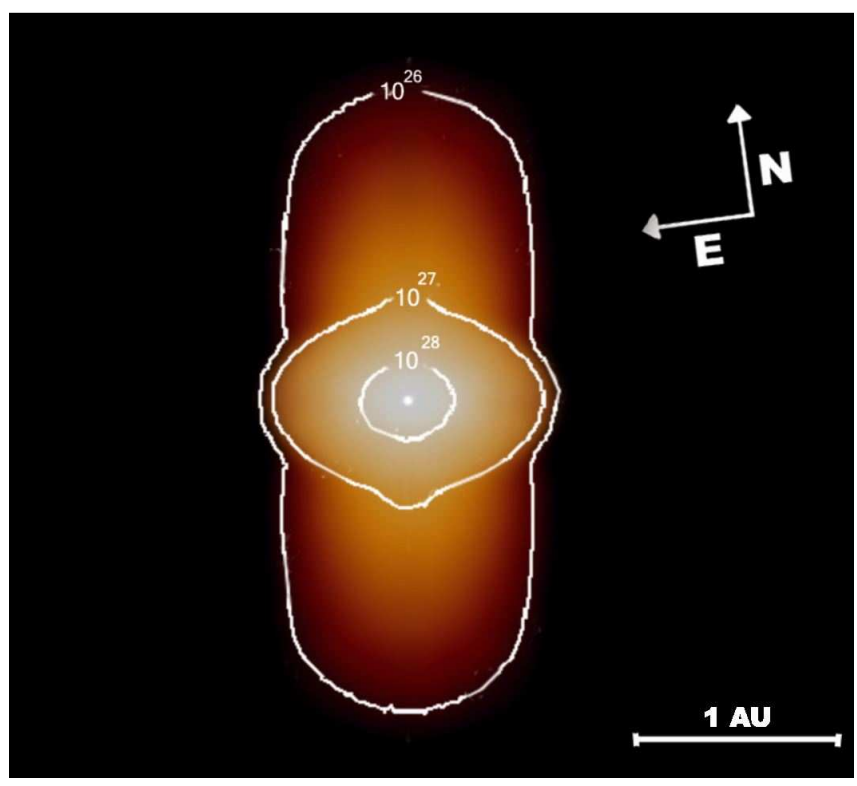

Figure 2. Intensity map in the continuum at $2.15 \mu \mathrm{m}$ obtained with SIMECA for the best model parameters of $\alpha$ Arae. The inclination angle is $55^{\circ}$, the central bright region is the flux contribution from the thin equatorial disk whereas the smoother regions originate from the stellar wind. The brightness contrast between the disk and the wind is globally $\sim 30$ but can reach 100 if you compare the inner region of the disk with the outer parts of the wind.

of the disk and to infer its rotation law (see Fig. 3). Meilland et al. ${ }^{7}$ obtained, for the first time, the direct evidence that the disk is in Keplerian rotation, answering a question that has existed since the discovery of the first Be star $\gamma$ Cas by Father Secchi in 1866. The disk around $\alpha$ Arae is compatible with a dense equatorial matter confined in the central region whereas a polar wind is contributing along the rotational axis of the central star. Between these two regions the density must be low enough to reproduce the large visibility amplitudes obtained for two of the four VLTI baselines.

Thanks to these first spectrally resolved interferometric measurements of a Be star at $2 \mu \mathrm{m}$ we were able to propose a possible scenario for the Be star $\alpha$ Arae circumstellar environment which consists of a thin disk and polar enhanced winds that is successfully modeled with the SIMECA code (see Fig. 2).

Using differential visibility amplitudes and phases across the $\operatorname{Br} \gamma$ line, Meilland et al. ${ }^{8}$ detected an asymmetry in the circumstellar structure around $\kappa$ Canis Majoris. However, $\kappa \mathrm{CMa}$ is difficult to fit within the classical scenario for Be stars, i.e. a fast rotating B star close to its breakup velocity surrounded by a Keplerian circumstellar disk with an enhanced polar wind. We found that $\kappa$ CMa does not seem to be a critical rotator, the rotation law within the disk is not Keplerian and the detected asymmetry seems to be hardly explained within the one-armed viscous disk framework.

The first high spatial and spectral observations of the circumstellar envelope of CPD-57²874, a B [e] supergiant, have been performed with the VLTI. Spectra, visibilities, and closure phase were obtained using the beam-combiner instruments AMBER and MIDI. ${ }^{9}$ In a first order analysis of the wavelength-dependent size and geometry of the circumstellar envelope of CPD- $57^{\circ} 2874$ we fitted the visibilities by an elliptical Gaussian model with diameters varying linearly with wavelength. Typical angular sizes of the major-axes are $3.4 \mathrm{mas}(2.2 \mu \mathrm{m}$ continuum from AMBER), 5.2 mas (Br $\gamma$ emission line from AMBER), and 15 mas (12 $\mu$ m continuum from MIDI). The spectro-interferometric VLTI observations and data analysis support the non-spherical, gaseous and dusty circumstellar envelope paradigm for B $[\mathrm{e}]$ supergiants. 

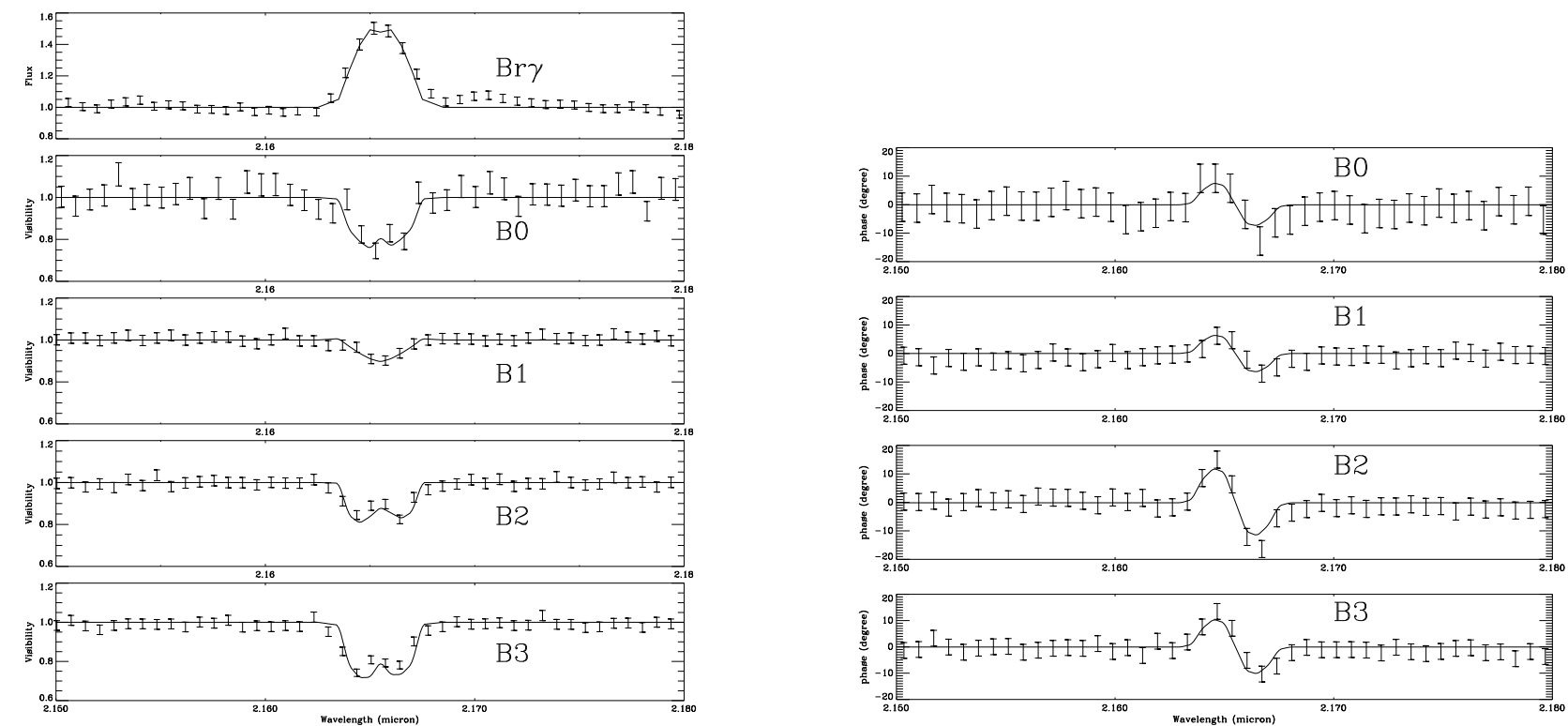

Figure 3. Relative visibility (left) and differential phases (right) of $\alpha$ Arae across the $\operatorname{Br} \gamma$ line profile for several VLTI baselines. The first picture from the top left is the $\operatorname{Br} \gamma$ line profile. The plain line are the fits we obtain with SIMECA from our best model whereas the VLTI/AMBER data are the points with error bars.

\section{MASS LOSS FROM MASSIVE STARS}

$\eta$ Car is one of the most luminous $\left(L \sim 4 \times 10^{6} L_{\odot}\right)$ and most massive $\left(M \sim 100 M_{\odot}\right)$ unstable Luminous Blue Variables suffering from an extremly high mass loss rate. Spectroscopic studies of the Homunculus nebula showed that the wind of $\eta$ Car is latitude-dependent. Van Boeckel et al. ${ }^{10}$ resolved the optically thick, aspheric wind region with NIR interferometry using the VLTI/VINCI instrument. This aspheric wind can be explained by models for line-driven winds from luminous hot stars rotating near their critical speed. The models predict a higher wind speed and density along the polar axis than in the equatorial plane. A variety of observations suggest that the central source of $\eta$ Car is a binary even if the binary nature of the central object in $\eta$ Car is still a matter of debate.

Our AMBER observations of $\eta \mathrm{Car}^{11}$ were performed with three Unit Telescopes and with spectral resolutions of $R=1,500$ (MR mode) and $R=12,000$ (HR mode) in Dec. $2004(\phi=0.268)$ and Feb. $2005(\phi=0.299)$ in spectral windows around the HeI and $\mathrm{Br} \gamma$ emission lines at $\lambda=2.059$ and $2.166 \mu \mathrm{m}$, respectively. From the measurements, we obtained spectra, visibilities, differential visibilities, differential phases, and closure phases. With projected baseline lengths up to $89 \mathrm{~m}$, an angular resolution of $\sim 5$ mas was achieved in the $K$ band.

In the $K$-band continuum, we resolved $\eta$ Car's optically thick wind. From a Gaussian fit of the $K$-band continuum visibilities in the projected baseline range from 28-89 m, we obtained a FWHM diameter of $4.0 \pm 0.2$ mas. When comparing the AMBER continuum visibilities with the NLTE radiative transfer model from Hillier et al., ${ }^{12}$ we find very good agreement between the model and observations. The best fit was obtained with a slightly rescaled version of the original Hillier et al. ${ }^{12}$ model, corresponding to an observed FWHM diameter of 2.4 mas and a $50 \%$ encircled-energy diameter of 4.3 mas at $\lambda=2.17 \mu \mathrm{m}$. Taking the different FOVs into account, we found good agreement between the AMBER measurements and previous VLTI/VINCI observations of $\eta$ Car presented by van Boeckel et al. ${ }^{10}$ If we fit Hillier et al. ${ }^{12}$ model visibilities to the observed AMBER emission line visibilities, we obtain $50 \%$ encircled-energy diameters of 6.5 and 9.6 mas in HeI and the Br $\gamma$ emission lines, respectively.

For both the $\operatorname{Br} \gamma$ and the HeI emission lines, we measured non-zero differential phases and non-zero closure phases within the emission lines, indicating a complex, asymmetric object structure. We developed a physically 


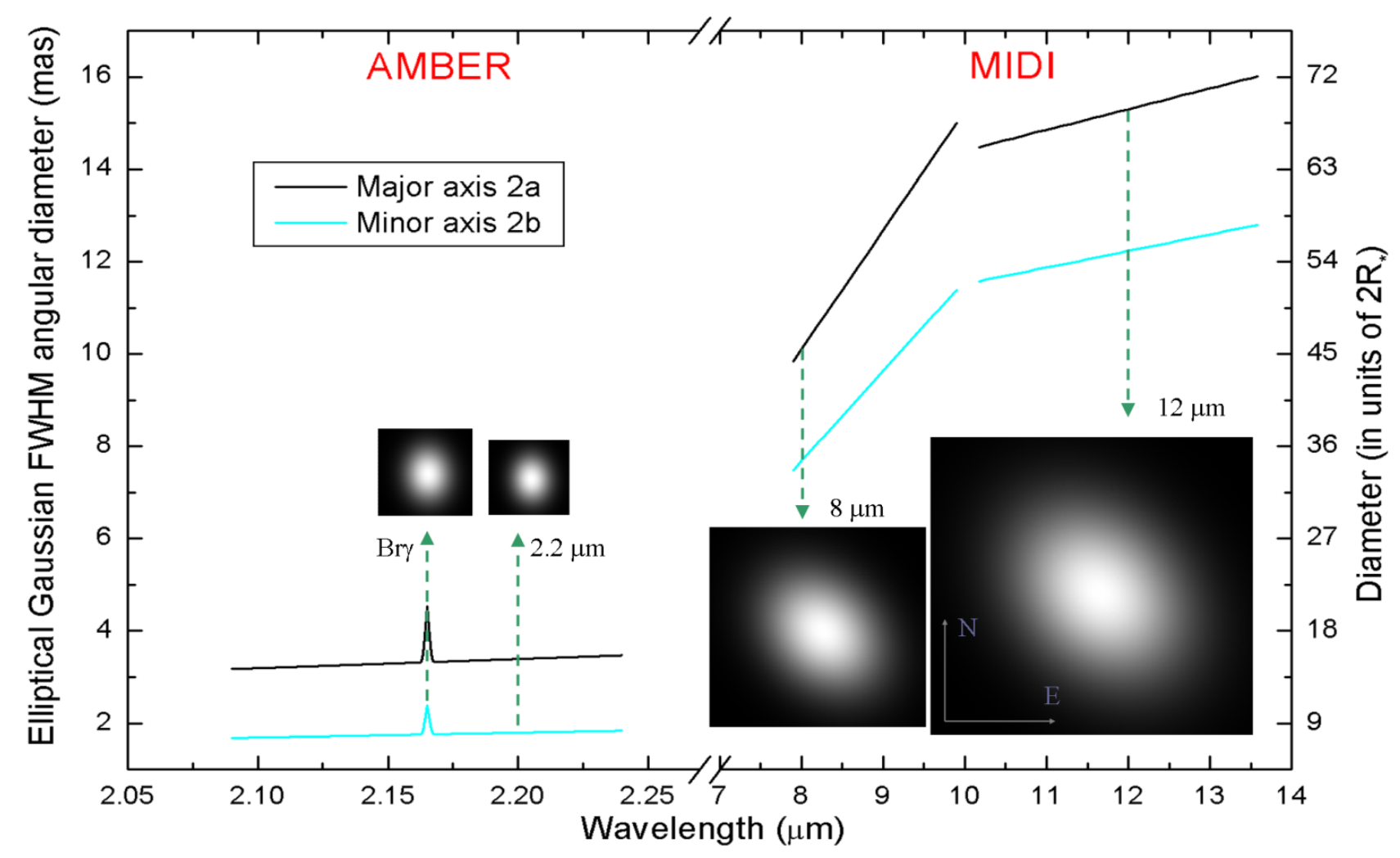

Figure 4. Wavelength-dependent sizes of the B[e] supergiant CPD-57 2874 derived from the fit of a chromatic elliptical Gaussian model to the VLTI/AMBER and VLTI/MIDI visibilities. The scale in the right is given in stellar diameters $\left(2 R_{*}\right)$, where the radius $R_{*}$ is estimated to be $60 \pm 15 R_{\odot}$.

motivated model, ${ }^{11}$ which shows that the asymmetries measured within the wings of the Br $\gamma$ line with differential and closure phases are consistent with the geometry expected for an aspherical, latitude-dependent stellar wind.

\section{INTERACTING BINARY IN LATE STELLAR EVOLUTION}

The Wolf-Rayet and $\mathrm{O}$ star binary system $\gamma^{2}$ Velorum has been investigated by AMBER. The goal was to understand better the wind of a WR star in an interacting binary on this double line spectroscopic system already observed with intensity interferometry. ${ }^{13}$ Strong signals were obtained in all interferometric measures, showing that the image of the system is strongly dominated by two compact, unresolved sources around the two stars location. The parameters of the binary have been accurately derived and have been shown to be stable with regard to the different approaches used to model the spectrum of the two components and to evaluate the contribution of different kinds of circumstellar material. In particular, Petrov et al. ${ }^{14}$ demonstrated that the combination of differential visibility, differential phase and closure phase as a function of $\lambda$ allows to obtain independantly the angular separation vector and the spectra of the components. Therefore, they have a new measure of the distance at $368_{-13}{ }^{+} 38 \mathrm{pc}$. This lies between the pre Hipparcos spectro-photometric estimations ( $450 \mathrm{pc}$ ) and the Hipparcos measure of $258 \mathrm{pc}$, yielding one more reason to consider revision of Hipparcos distances of complex sources close to the performance limit. A second result of the work is a direct, model independent, measurement of the spectrum of the WR component which opens the possibility of improved modelling of this star. Whatever the fit of the data with a binary system, the measures display, in tens of spectral channels, a $5 \sigma$ to $10 \sigma$ residual which is the signature of circumstellar material. The dust contribution to the continuum is limited to less than $5 \%$ of the total flux. The residuals exhibit spectral features which allow to speculate that they result from interstellar gas, probably near the wind-wind collision zone, contributing to the emission lines and also to the continuum with free-free emission. ${ }^{14}$ 


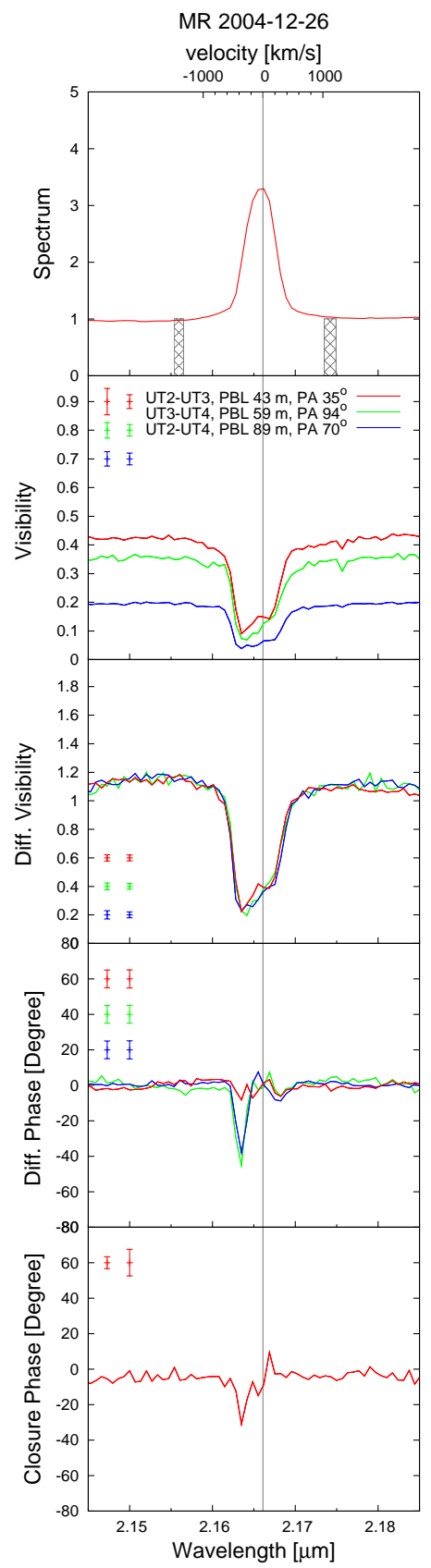

Figure 5. AMBER observables derived from our $\eta$ Car data around the Br $\gamma$ line (MR mode, 2004 December 26; the HR mode and He I observations are described in Weigelt et $\mathrm{al}^{11}$ ). The top panel shows the spectrum as extracted from the interferometric channels, followed by the derived calibrated visibilities and the differential visibilities. In the two panels at the bottom, the differential phase and the closure phase are presented. The vertical grey line marks the rest-wavelength of $\operatorname{Br} \gamma\left(\lambda_{\text {vac }}=2.1661 \mu \mathrm{m}\right)$. The left error bars correspond to the total error estimated for the continuum wavelength range, and the error bars towards the right visualizes the total error for the wavelength range within the line. 


\section{CONCLUSION}

The first astrophysical results from AMBER have focused on stellar wind structure, kinematics, and its interaction with dust usually concentrated in a disk. Because AMBER has dramatically increased the number of measures per baseline, this instrument brings strong constraints on morphology and models despite a relatively poor $(u, v)$ coverage for each object.

From these initial measurements, we can deduce that there might be an ubiquity of an equatorial disk of dust and/or gas with a latitude dependant wind. We can wonder if this results from a selection effect, a term bias or an actual breakthrough. For other observing programs the reader can read the AMBER science program ${ }^{4}$ described in last SPIE conference.

The VLTI is currently limited by vibrations and is under intensive tests. Hopefully, the consolidation plan undertaken by ESO will soon allow us to achieve the ultimate performance of AMBER.

\section{ACKNOWLEDGMENTS}

We would like to thanks all persons who made it possible to conceive, build and install the AMBER instrument.

\section{REFERENCES}

1. R. G. Petrov, F. Malbet, G. Weigelt, F. Lisi, P. Puget, P. Antonelli, U. Beckmann, S. Lagarde, E. Lecoarer, S. Robbe-Dubois, G. Duvert, S. Gennari, A. Chelli, M. Dugue, K. Rousselet-Perraut, M. Vannier, and D. Mourard, "Using the near infrared VLTI instrument AMBER," in Interferometry for Optical Astronomy II. Edited by Wesley A. Traub . Proceedings of the SPIE, Volume 4838, pp. 924-933 (2003)., pp. 924-933, Feb. 2003.

2. R. G. Petrov and The AMBER Consortium, "Introducing the near infrared VLTI instrument AMBER to its users," Ap\&SSS 286, pp. 57-67, 2003.

3. F. Malbet, T. Bloecker, R. Foy, D. Fraix-Burnet, P. Mathias, A. Marconi, J. Monin, R. G. Petrov, P. Stee, L. Testi, and G. Weigelt, "Astrophysical potential of the AMBER/VLTI instrument," in Interferometry for Optical Astronomy II. Edited by Wesley A. Traub. Proceedings of the SPIE, Volume 4838, pp. 917-923 (2003)., pp. 917-923, Feb. 2003.

4. F. Malbet, T. M. Driebe, R. Foy, D. Fraix-Burnet, P. Mathias, A. Marconi, J.-L. Monin, R. G. Petrov, P. Stee, L. Testi, and G. P. Weigelt, "Science program of the AMBER consortium," in New Frontiers in Stellar Interferometry, Proceedings of SPIE Volume 5491. Edited by Wesley A. Traub. Bellingham, WA: The International Society for Optical Engineering, 2004., p.1722, W. A. Traub, ed., pp. 1722-+, Oct. 2004.

5. F. Malbet, M. Benisty, W. J. De Wit, S. Kraus, A. Meilland, F. Millour, E. Tatulli, J. . Berger, O. Chesneau, K. . Hofmann, A. Isella, A. Natta, R. Petrov, T. Preibisch, P. Stee, L. Testi, G. Weigelt, and AMBER Collaboration, "Disk and wind interaction in the young stellar object MWC 297 spatially resolved with VLTI/AMBER," $A \& A$ in press, pp. (eprint: astro-ph/051035), Oct. 2005.

6. E. Tatulli, A. Isella, A. Natta, L. Testi, A. Marconi, F. Malbet, P. Stee, R. Petrov, F. Millour, A. Chelli, G. Duvert, and AMBER Collaboration, "Constraining the wind lauching region in herbig ae stars: AMBER/VLTI spectroscopy of HD 104237," A\& A submitted, 2006.

7. A. Meilland, P. Stee, M. Vannier, F. Millour, A. Domiciano De Souza, F. Malbet, C. Martayan, F. Paresce, R. Petrov, A. Richichi, A. Spang, and the AMBER Consortium Collaboration, "First direct detection of a Keplerian rotating disk around the Be star $\$ \backslash$ alpha $\$$ Arae using the VLTI/AMBER instrument," $A \mathscr{E} A$ in press, pp. (eprint: astro-ph/060640), June 2006.

8. A. Meilland, F. Millour, P. Stee, A. Domiciano de Souza, R. Petrov, D. Mourard, S. Jankov, S. RobbeDubois, A. Spang, and AMBER Collaboration, "An asymmetry detected in the disk of $\kappa$ CMa with AMBER/VLTI," A\&\&A submitted, 2006.

9. A. Domiciano de Souza, T. Driebe, O. Chesneau, K. Hofmann, S. Kraus, A. S. Miroshnichenko, K. Ohnaka, R. G. Petrov, T. Preibisch, P. Stee, G. Weigelt, F. Lisi, F. Malbet, and A. Richichi, "VLTI/AMBER and VLTI/MIDI spectro-interferometric observations of the B[e] supergiant CPD-57 2874," $A \mathscr{E} A$ in press, pp. (eprint: astro-ph/0510735), Oct. 2005. 
10. R. van Boekel, P. Kervella, M. Schöller, T. Herbst, W. Brandner, A. de Koter, L. B. F. M. Waters, D. J. Hillier, F. Paresce, R. Lenzen, and A.-M. Lagrange, "Direct measurement of the size and shape of the present-day stellar wind of eta Carinae," A\&A 410, pp. L37-L40, Oct. 2003.

11. G. Weigelt, T. Kraus, S. Driebe, R. Petrov, K. Hofmann, O. Millour, F. Chesneau, D. Schertl, F. Malbet, J. Hillier, T. Gull, K. Davidson, A. Domiciano de Souza, and AMBER Collaboration, "Near-infrared interferometry of $\eta$ Carinae with high spatial and spectral resolution using the VLTI and the AMBER instrument," A\&BA submitted, 2006.

12. D. J. Hillier, K. Davidson, K. Ishibashi, and T. Gull, "On the Nature of the Central Source in $\eta$ Carinae," ApJ 553, pp. 837-860, June 2001.

13. R. Hanbury Brown, J. Davis, D. Herbison-Evans, and L. R. Allen, "A study of $\gamma^{2}$ Velorum with a stellar intensity interferometer.," MNRAS 148, pp. 103-117, 1970.

14. R. Petrov, F. Millour, O. Chesneau, D. Bonneau, L. Dessart, C. Bechet, I. Tallon-Bosc, M. Tallon, E. Thiébaut, F. Vakili, F. Malbet, D. Mourard, and AMBER Collaboration, "Direct constraints on the distance of $\gamma^{2}$ Velorum based on AMBER/VLTI observations," A\&A submitted, 2006. 